\title{
Dietary ecology of the extinct cave bear: Evidence of omnivory as inferred from dental microwear textures
}

\author{
D. Brent Jones and Larisa R.G. DeSantis \\ Acta Palaeontologica Polonica 61 (4), 2016: 735-741 doi:http://dx.doi.org/10.4202/app.00253.2016
}

The diet of the extinct European cave bear, Ursus spelaeus, has widely been debated. Diverging from the extant brown bear (Ursus arctos) approximately 1.2 million years ago, the cave bear is one of the most ubiquitous fossil bears occurring in Europe during the middle and Late Pleistocene. Early morphological studies suggested that the cave bear was likely specialized on processing tough and/or abrasive foods, while later two-dimensional low-magnification microwear studies suggested that they were omnivorous and may have consumed more bone than $U$. arctos. Here, we used dental microwear texture analysis (DMTA) to further interpret the diet of the cave bear. Microscopic wear features were assessed and compared to modern ursids, including the cave bears' closest living relative, $U$. arctos. Results suggest that $U$. spelaeus consumed a diet with a diversity of textural properties, similar to most other bears and only distinguishable from the hyper-carnivorous polar bear (Ursus maritimus). Further, only $U$. maritimus can be distinguished from all bear species here examined (i.e., the giant panda bear, Ailuropoda melanoleuca; sun-bear, Ursus malayanus; spectacled bear, Tremarctos ornatus; American black bear, Ursus americanus; and U. arctos), with significantly greater area-scale fractal complexity (Asfc) of microwear surfaces. The DMTA of A. melanoleuca also has significantly lower Asfe than T. ornatus and U. americanus, consistent with observed dietary behavior. As modern bears vary their diets seasonally and across their range, it may be difficult to characterize the dietary ecology of extinct bears using dental microwear alone. Nevertheless, DMTA here demonstrates that $U$. spelaeus had a diet distinct from the hyper-carnivorous $U$. maritimus and instead likely consumed food with textural properties most similar to other herbivorous/omnivorous bears. Lastly, the European cave bear and North American giant short-faced bear (Arctodus simus) may have had similar diets as evident from DMTA, with $U$. spelaeus potentially eating tougher food items.

Key words: Mammalia, Ursus spelaeus, carnivore, dental microwear, Pleistocene, Europe.

Brent Jones [davis.b.jones@vanderbilt.edu] and Larisa R.G. DeSantis [Larisa.desantis@vanderbilt.edu] (corresponding author), Department of Earth and Environmental Science, Vanderbilt University, Nashville, Tennessee 37235, USA. 
This is an open-access article distributed under the terms of the Creative Commons

Attribution License (for details please see creativecommons.org), which permits unrestricted use, distribution, and reproduction in any medium, provided the original author and source are credited.

\author{
FoF \\ Far) Supplementary file $(129.8 \mathrm{kB})$
}

